\title{
FATIGUE FAILURE IN THIN-FILM POLYSILICON IS DUE TO SUBCRITICAL CRACKING WITHIN THE OXIDE LAYER
}

D. H. Alsem, ${ }^{\text {a),b),c) }}$ E. A. Stach, ${ }^{\text {b),c) C. L. Muhlstein, }{ }^{\text {d) }} \text { and R. O. Ritchie }}{ }^{\text {a),b) }}$

a) Department of Materials Science and Engineering, University of California, Berkeley, California 94720

b) Materials Sciences Division, Lawrence Berkeley National Laboratory, Berkeley, California 94720

c) National Center for Electron Microscopy, Lawrence Berkeley National Laboratory, Berkeley, California 94720

d) Department of Materials Science and Engineering, Pennsylvania State University, University Park, Pennsylvania 16802

It has been established that microelectromechanical systems (MEMS) created from polycrystalline silicon thin-films are subject to cyclic fatigue. Prior work by the authors has suggested that although bulk silicon is not susceptible to fatigue failure in ambient air, fatigue in micron-scale silicon is a result of a "reaction-layer" process, whereby high stresses induce a thickening of the post-release oxide at stress concentrations such as notches, which subsequently undergoes moisture-assisted cracking. However, there exists some controversy regarding the post-release oxide thickness of the samples used in the prior study. In this Letter, we present data from devices from a more recent fabrication run that confirm our prior observations. Additionally, new data from tests in high vacuum show that these devices do not fatigue when oxidation and moisture are suppressed. Each of these observations lends credence to the 'reaction-layer' mechanism. 
Recent advances in the design of microelectromechanical systems (MEMS) have increased the demand for more reliable microscale structures. ${ }^{2}$ Although silicon is an effective and widely used structural material at the microscale, it is very brittle. Consequently, reliability is a limiting factor for commercial and defense applications. Since the surface to volume ratio of these structural films is very large, classical models for failure modes in bulk materials cannot always be applied. For example, whereas bulk silicon is immune to cyclic fatigue failure ${ }^{3,4}$ thin micron-scale structural films of silicon appear to be highly susceptible. ${ }^{1,5-14}$ It is clear that at these size scales, surface effects may become dominant in controlling mechanical properties.

The main reliability issues for MEMS are stiction, fatigue and wear. ${ }^{15}$ Fatigue is important in cases where devices are subjected to a large number of loading cycles with amplitudes below their (single-cycle) fracture stress, which may arise due to vibrations intentionally induced in the structure (i.e. a resonator) or those which arise from the service environment. While the reliability of MEMS has received extensive attention ${ }^{16,17}$, the physical mechanisms responsible for these failure modes have yet to be conclusively determined. This is particularly true for fatigue, where the mechanisms have been subject to intense debate. ${ }^{1,7-9,11,14}$ Recently we have proposed that the fatigue of micron-scale polysilicon is associated with stress-induced surface oxide thickening and moistureassisted subcritical cracking in the amorphous $\mathrm{SiO}_{2}$ oxide layer ("reaction-layer" fatigue). ${ }^{1,7-8,18}$ The mechanism of oxide thickening is as yet unknown, but is likely related to some form of stress-assisted diffusion. Allameh et al ${ }^{11}$ suggest a complementary mechanism involving stress-assisted oxide thickening, caused by dissolution of the surface oxide which forms deep grooves that are sites for crack initiation. Kahn et al $l^{9,14}$ 
have criticized these mechanisms and proposed that, instead, fatigue is caused by subcritical cracking due to contacting surface asperities in the compressive part of the cycle. To the authors' knowledge, there is no direct experimental observation of such asperity contact. Also, their model cannot explain why micron-scale silicon, and not bulk silicon, is susceptible to fatigue. Moreover, Kahn et $a l^{9,14}$ do not acknowledge the role of stress-induced oxide thickening, which has been observed directly using TEM and indirectly using atomic-force microscope measurements by several investigators, $1,8,10,11$ and have questioned whether the materials utilized by Muhlstein et al. ${ }^{1,8}$ and Allameh et $a l .{ }^{11}$ were representative due to the relatively thick oxide scales.

Accordingly, the goal of the present research is to seek a definitive understanding of the physical mechanisms responsible for fatigue in polysilicon structural thin-films. Our approach is to combine on-chip testing methods with electron microscopy by fatiguing thin-film samples and observing them, in an unthinned condition, using high-voltage transmission electron microscopy (HVTEM). Two principal results are found from this work: (i) fatigue tests conducted in ambient air on polysilicon samples from a more recent fabrication run confirm that the fatigue behavior and oxide-layer thicknesses observed in earlier experiments ${ }^{1,8,10,11}$ were not an artifact of that particular fabrication run, and more importantly, (ii) fatigue tests run in high vacuum reveal absolutely no evidence of premature fatigue failures. We believe that both of these results add further confirmation to the "reaction-layer" mechanism for fatigue of micron-scale polysilicon.

Phosphorous-doped $\left(\mathrm{n}^{+}\right.$-type) polysilicon fatigue resonator devices (electrostatically actuated by an interdigited comb-drive (Figure 1a) in $\sim 25^{\circ} \mathrm{C}, 30-50 \%$ relative humidity (RH) air at $\sim 40 \mathrm{kHz}$ with a load ratio (ratio of minimum to maximum load) of $R=-1$ ) 
have been used to measure the fatigue behavior of notched cantilever beams (Figure 1b). The second comb drive is used to monitor the displacement using capacitive sensing electronics. Corresponding finite-element calculations permit the determination of the stresses at the notch root. ${ }^{6,7}$ In prior work, "metal-like" fatigue behavior, in the form of an applied stress versus number of cycles to failure $(S / N)$ curve, was obtained for $2-\mu \mathrm{m}$ thick polysilison. ${ }^{1}$ Additionally, HVTEM of unthinned fatigue test devices revealed a thickening of the post-release oxide layer at the notch root for fatigued samples (in contrast to manually fractured specimens which showed no such thickening); moreover, small $(\sim 30-50 \mathrm{~nm})$ cracks were observed in this oxide layer of fatigued samples ${ }^{8}$, consistent with in situ changes in the natural frequency of the test device. ${ }^{18}$ This led us to propose a "reaction-layer" mechanism for the fatigue of thin-film polysilicon (Figure 2): (i) the initial post-release oxide layer grows while mechanically stressed during cycling, (ii) stress/moisture-assisted cracking of the oxide layer results in stable crack growth, assisted by the well-known vulnerability of amorphous $\mathrm{SiO}_{2}$ to environmentally-induced cracking, where hydroxyl ions in water react chemically with the $\mathrm{SiO}_{2}$, destroying the siloxane bonds ${ }^{19}$ and (iii) provided the oxide layer is thick enough, the critical crack size for the entire structure is reached inside the oxide layer and the structure fails catastrophically.

In this Letter, we report on fatigue data acquired from devices from a different fabrication run at the Multi User MEMS Process (MUMPs) foundry, as well as new observations from tests in high vacuum. In light of the ongoing discussion on the mechanism causing fatigue in thin-film silicon ${ }^{1,7-9,11,13}$, the rationale for this work is to check the reproducibility of our previous findings across fabrication runs (i.e., fatigue 
behavior and oxide-layer thicknesses) and to characterize the changes in fatigue behavior and development of reaction layers in a relatively oxygen and moisture-free environment. Figure 3 shows the $S / N$ curve from previously obtained fatigue data in ambient air from devices from MUMPs run 18 (best fit line). ${ }^{1,7-8}$ These results are compared to the current data from MUMPs fabrication run 50, acquired in both ambient air $\left(\sim 25^{\circ} \mathrm{C}, 30-40 \% \mathrm{RH}\right)$ and high vacuum $\left(\sim 2.0 \times 10^{-7} \mathrm{mbar} \sim 25^{\circ} \mathrm{C}\right)$. It is apparent that the new results in ambient air closely follow those obtained previously on an earlier MUMPs run; more importantly, no samples failed in vacuo, even at high stresses after cycling in excess of $10^{9}-10^{10}$ cycles (note: all vacuum data points are plotted at the point where the tests were stopped - no device failure occurred despite the large number of loading cycles).

In order to image the oxide in the notch root, the samples that did not fatigue in vacuo were either fractured manually or by applying large displacements electrostatically. Thereafter, they were lifted from the chip onto a copper clamshell grid for TEM examination. Because of the high acceleration voltage $(800 \mathrm{kV})$ used, no thinning of the $2-\mu \mathrm{m}$ thick specimens was necessary. HVTEM images of fatigued and fractured devices in ambient air and high vacuum reveals a thickening of the oxide layer up to $100 \mathrm{~nm}$ for specimens fatigued in ambient air (Figure 4a). However, this local thickening at the notch root was not observed for fractured (non-fatigued) specimens in ambient air (Figure 4b), or for specimens subjective to fatigue attempts in vacuum (Figure $4 \mathrm{c}$ - oxide layers here ranged from $\sim 15-30 \mathrm{~nm}$ ). For the latter specimens, clearly the absence of moisture in high vacuum prevents subcritical crack growth in the post release oxide layer; moreover, as this layer does not exceed $30 \mathrm{~nm}$, it is not large enough to accommodate the critical crack size for high cycle failure of the entire structure, which is $\sim 50 \mathrm{~nm}^{18}$. Even if the 
stresses are high, i.e., slightly below the fracture strength, and the critical crack size would be small enough to occur inside the unthickened oxide layer, the device does not fail in vacuum. This suggests that there is no stable subcritical crack propagation in the absence of moisture and oxygen.

Both the newly acquired fatigue data in air and high vacuum, as well as the TEM micrographs of the oxide layers, provide further evidence that "reaction layer" fatigue is the governing mechanism for fatigue failure in micron-scale polycrystalline silicon thinfilms.

This work was funded by the Director, Office of Science, Office of Basic Energy Sciences, Division of Materials Sciences and Engineering, of the U.S. Department of Energy under Contract No. DE-AC03-76SF00098. 
1. C. L. Muhlstein, E. A. Stach, and R. O. Ritchie, Appl. Phys. Lett. 80, 1532 (2002).

2. M. J. Madou, Fundamentals of Microfabrication, 2nd ed. (CRC Press, Boca Raton, 2002).

3. R. O. Ritchie, Int. J. Fract. 100, 55 (1999).

4. S. Suresh, Fatigue of Materials, $2^{\text {nd }}$ ed. (Cambridge University Press, Cambridge, 1998).

5. J. A. Connally and S. B. Brown, Science 256, 1537 (1992).

6. C. L. Muhlstein, S. B. Brown, and R. O. Ritchie, J. Microelectromech. Syst. 10, 593 (2001).

7. C. L. Muhlstein, S. B. Brown, and R. O. Ritchie, Sens. Actuators A 94,177 (2001).

8. C. L. Muhlstein, E. A. Stach, and R. O. Ritchie, Acta. Mater. 50, 3579 (2002).

9. H. Kahn, R. Ballerini, J. J. Bellante, and A. H. Heuer, Science 298, 1215 (2002).

10. O. Millet, B. Legrand, D. Collard, and L. Buchaillot, Jpn. J. Appl. Phys., Part 2 (Letters), 41(11B), 1339 (2002).

11. S. M. Allameh, P. Shrotriya, A. Butterwick, S. B. Brown and W. O. Soboyejo, J. Microelectromech. Syst. 12, 313 (2003).

12. J. Bagdahn and W. N. Sharpe Jr., Sens. Actuators A 103, 9 (2003).

13. X. Li and B. Bhushan, Surface and Coatings Technology 163-164, 521 (2003).

14. H. Kahn, R. Ballerini, and A. H. Heuer, Current Opinion in Solid State and Materials Science, (2004), 8, 71 (2004).

15. A. D. Romig Jr., M. T. Dugger and P. J. McWhorter, Acta. Mater. 51, 5837 (2003).

16. C. L. Muhlstein and S.B. Brown, eds. Mechanical Properties of Structural Films (STP 1413), American Society for Testing and Materials: West Conshohocken, PA. (2001) pp. 333.

17. S. Brown, J. Gilbert, H. Guckel, R. Howe, G. Johnson, P. Krulevitch, and C.

Muhlstein, eds. Microelectromechanical Structures for Materials Research. Vol. 518, (Materials Research Society: San Francisco, CA. 1998).

18. C. L. Muhlstein and R. O. Ritchie, Int. J. Fracture 119 (4), 449 (2003).

19. S. M. Wiederhorn, J. Am. Chem. Soc. 55, 81 (1972). 


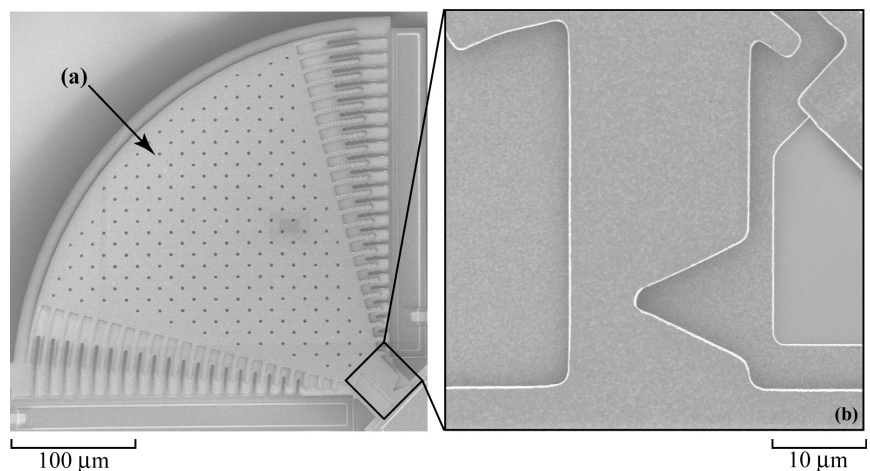

Figure 1: 

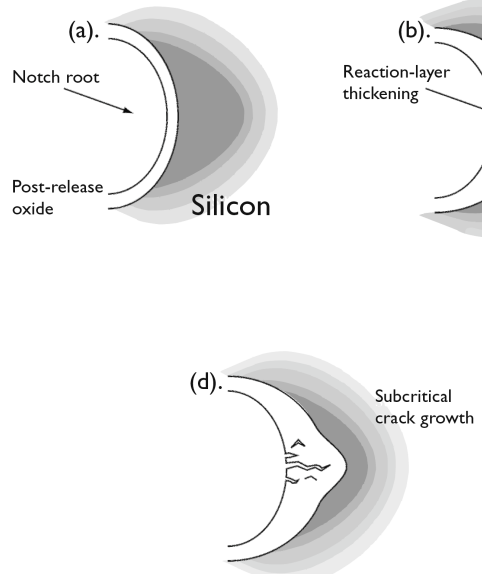
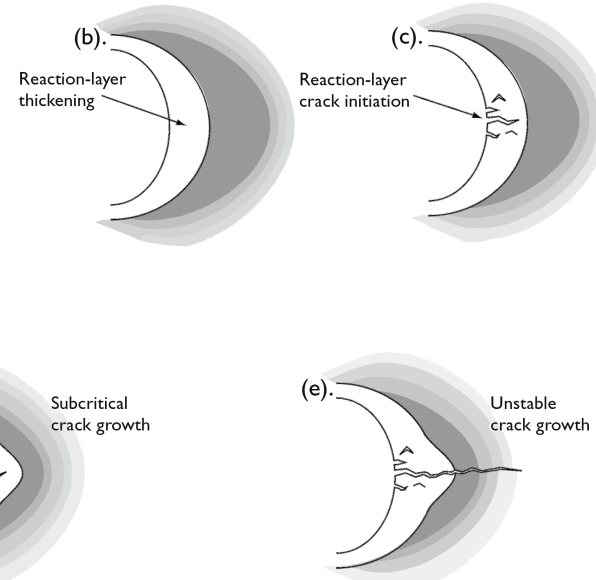

Figure 2: 


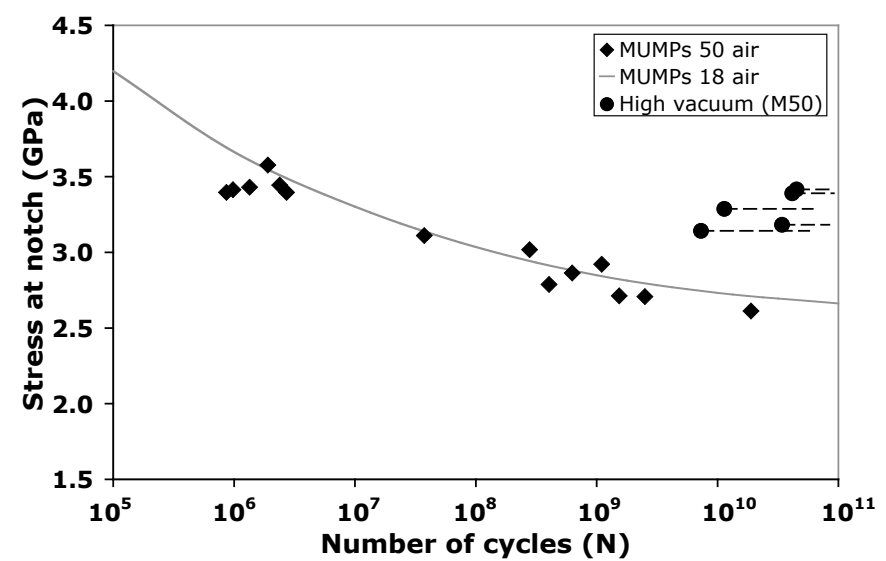

Figure 3: 

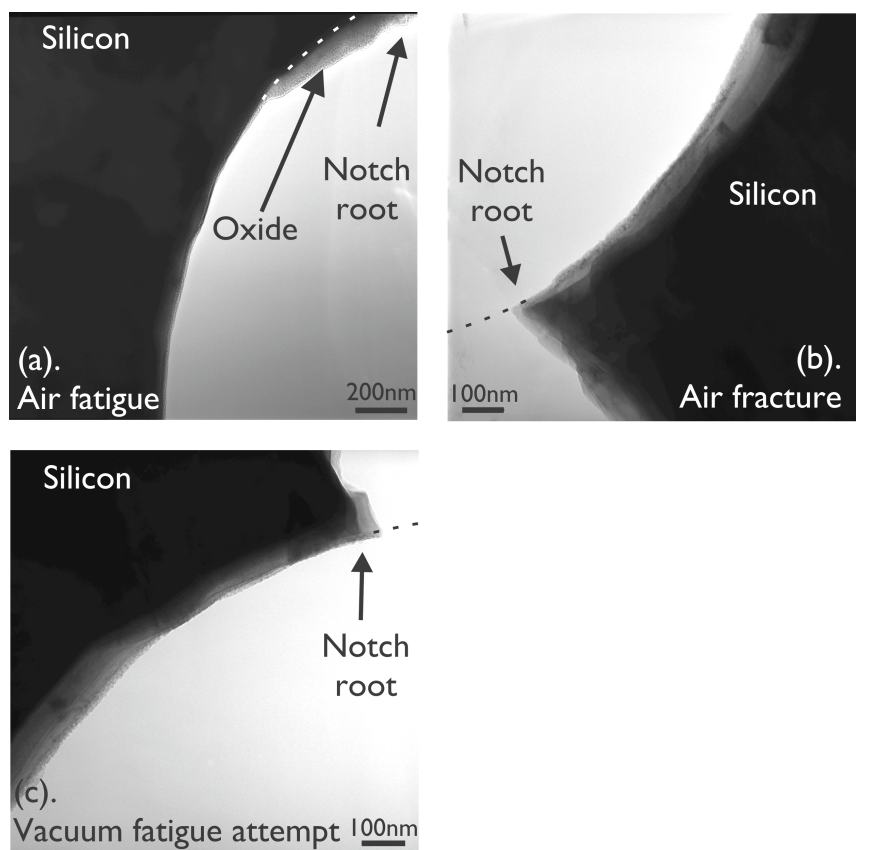

Figure 4 
Figure 1: Scanning electron micrographs of the polysilicon MEMS fatigue life characterization resonator fabricated at the MEMSCAP MUMPs foundry (formerly CRONOS http://www.memscap.com/memsrus). (a) Triangular proof mass (thickness 2 $\mu \mathrm{m})$ with interdigited comb drive electrostatical actuator and capacitive displacement sensor comb; (b) notched cantilever beam connecting the resonator mass and anchor.[C. L. Muhlstein et al, Appl. Phys. Lett. 80, 1532 (2002)]

Figure 2: Schematic of the "reaction-layer" fatigue mechanism at the notch of the polycrystalline silicon cantilever beam (a). (b): Localized oxide thickening at the notch root. (c): Environmentally-assisted crack initiation in the native oxide at the notch root. (d): Additional thickening and cracking of reaction layer. (e): Unstable crack growth in the silicon film. [C. L. Muhlstein et al, Appl. Phys. Lett. 80, 1532 (2002)]

Figure 3: Polysilicon $\mathrm{S} / \mathrm{N}$ curve. Fitted line for previous data from MUMPs run 18 in ambient air compared with fatigue data from MUMPs run 50 in ambient air and in vacuo $\left(\sim 2 \times 10^{-7} \mathrm{mbar}\right)$.

Figure 4: HVTEM images from failed resonator devices.

(a): Fatigued in ambient air with thickened oxide layer around the notch root (principal stress at the notch root: $2.86 \mathrm{GPa}$; number of cycles at failure: $6.28 \times 10^{8}$ ).

(b): Manually fractured specimen in ambient air: no (local) oxide thickening. Because of sample tilt, some contrast in grains at the edge is visible. Only the top transparent part is amorphous.

(c): Device after fatigue attempt in high vacuum and manual fracture: no oxide layer thickening (principal stress at the notch root during fatigue attempt: $3.29 \mathrm{GPa}$, number of cycles when stopped: $1.14 \times 10^{10}$ ). Also in this case contrast from grains on the edge is 
visible because of sample tilt and therefore only the top amorphous layer is oxide 